\title{
Lesueur. Gouaches révolutionnaires.
}

\section{Annie Duprat}

\section{(2) OpenEdition}

\section{Journals}

Édition électronique

URL : https://journals.openedition.org/ahrf/1960

DOI : 10.4000/ahrf.1960

ISSN : 1952-403X

Éditeur :

Armand Colin, Société des études robespierristes

Édition imprimée

Date de publication : 1 décembre 2005

Pagination : 249-250

ISSN : 0003-4436

\section{Référence électronique}

Annie Duprat, «Lesueur. Gouaches révolutionnaires. ». Annales historiques de la Révolution française [En ligne], 342 I octobre-décembre 2005, mis en ligne le 05 avril 2006, consulté le 23 avril 2022. URL : http://journals.openedition.org/ahrf/1960 ; DOl : https://doi.org/10.4000/ahrf.1960

Ce document a été généré automatiquement le 23 avril 2022.

Tous droits réservés 


\title{
Lesueur. Gouaches révolutionnaires.
}

\author{
Annie Duprat
}

\section{RÉFÉRENCE}

Philippe de Carbonnières, Lesueur. Gouaches révolutionnaires. Collections du Musée Carnavalet, Paris, Paris-Musées et éditions Nicolas Chaudun, 2005, 255 p., ISBN

2-87900-858-1, $55 €$

1 Qui n'a pas un jour croisé, dans un manuel scolaire, un livre d'histoire ou sur une affiche l'une de ces scènes finement dessinées et coloriées, découpées puis collées sur des fonds d'un bleu si apaisant qu'elles en vous feraient oublier la «tourmente révolutionnaire » qu'elles représentent ? Les gouaches de Lesueur sont très célèbres : mais sont-elles connues ? Déposées par le Musée du Louvre au Musée Carnavalet, qui en possède en propre quelques-unes, elles sont habituellement exposées avec parcimonie étant donné leur fragilité. Or, à l'occasion d'une exposition récente, Au temps des Merveilleuses: la société parisienne sous le Directoire et le Consulat, un accrochage exceptionnel des 64 gouaches de Lesueur a été effectué dans la galerie reliant l'Hôtel Carnavalet à l'Hôtel Le Pelletier de St-Fargeau, accompagné de cet ouvrage somptueux. Son auteur, Philippe de Carbonnières, responsable des collections révolutionnaires au Musée Carnavalet, a repris la totalité du « dossier Lesueur »; il nous livre le fruit de ses recherches ainsi que la reproduction (techniquement très réussie) des 64 gouaches exposées. La série pose en effet un grand nombre de questions, dont certaines n'ont pas encore trouvé de réponse. Par exemple, combien de gouaches ont été composées et pour quel(s) usage(s)? Qui est l'auteur des commentaires manuscrits qui les accompagnent? À quelle date, même approximative, ces images et ces textes ont-ils été composés? Qui se cache sous le nom de Lesueur? À cette question, Philippe de Carbonnières fournit une information des plus importantes. En effet, alors que l'hypothèse d'un auteur double, les frères Pierre-Étienne et Jacques-Philippe Lesueur était la plus communément admise, Philippe de Carbonnières nous invite à le suivre dans son examen de l'adresse parisienne des propriétaires de la collection, qui n'a pas changé depuis 1777 et qui est très proche de celle de l'imagier Jean-Baptiste Lesueur 
(1749-1826), membre du comité révolutionnaire de sa section (dont il a voulu démissionner en 1794) ce qui témoigne d'une raisonnable activité civique dans son quartier. Il figure sur une liste de terroristes en 1801. Cette information sur l'appartenance d'un Lesueur au comité révolutionnaire de sa section, alors que l'écriture de l'histoire de la Révolution qu'il fait sur ses gouaches est assez mesurée, n'est pas sans rappeler celles que nous avons évoquée dans le cas de Villeneuve, graveur et marchand d'estampes rue Zacharie-St Séverin à Paris, que les frères Goncourt et Renouvier considéraient comme un enragé en se fiant à l'observation de ses caricatures les plus féroces (voir «Autour de Villeneuve, le mystérieux auteur de la gravure La contre-révolution ", Annales historiques de la Révolution française, 1997, n 3, pp. 423-439). Il est toujours difficile de poser la question de l'engagement dans le cas d'artistes, qui sont aussi des marchands, dont on connaît peu de choses. Enfin, JeanBaptiste Lesueur exerce longtemps son activité à la même adresse comme l'indiquent les almanachs du commerce.

2 Mais, malgré une enquête iconographique de grande qualité, des zones d'ombre persistent. On ne sait pas encore combien de gouaches ont été faites. Il en existe aujourd'hui 83, dont 64 sont conservées à Carnavalet, les 19 autres appartenant à des héritiers de la famille Bidault de l'Isle. La numérotation qui figure sur les planches permettrait d'en évaluer le nombre à 94, mais, outre que la question de l'usage de cette numérotation n'a pas encore pu être élucidée, on peut supposer qu'il y en aurait eu davantage. En effet, il manque des scènes importantes, comme la prise de la Bastille, alors que figure sa démolition... Un autre argument peut être soulevé : celui de la numérotation des scènes car, aux numéros visibles sur les gouaches, et dont l'ordre a été conservé, tant pour le catalogue que pour l'accrochage, il faudrait en mentionner d'autres, qui figurent au dos et qui n'ont été repérés que lors des travaux de restauration. Enfin, la date des événements figurés, qui va jusqu'à un "songe de Napoléon » juste après Marengo (1807), pose question, car il y a un grand déséquilibre de sujets entre la période révolutionnaire jusqu'à Thermidor et des thématiques plus difficiles à dater (comme pour la planche 19, "Fabrication de fusils", ou encore la planche 52, "Misère et pénurie »). Cette question de la datation est encore compliquée par l'adjonction d'une série de textes manuscrits, collés au bas de chacune des gouaches, comme autant de commentaires, ironiques et complices le plus souvent, mais aussi moralisateurs et parfois critiques. Rappelons que de nombreuses caricatures de la collection Hennin comportent des mentions manuscrites de ce type, des commentaires qui sont à la fois des aide-mémoire et des jugements souvent désabusés sur l'événement représenté. On peut rejoindre Philippe de Carbonnières lorsqu'il formule l'hypothèse d'un décalage chronologique entre l'image et l'écrit, mais aussi l'idée que les gouaches auraient pu être réalisées tout au long de la période et sans doute de manière privilégiée après Thermidor. Il se fonde sur l'observation de la planche «Motion au Palais-Royal » qui présente des discordances entre la date de l'événement (juillet 1789) et les costumes (plutôt de l'époque directoriale). Nous touchons ici à l'une des difficultés majeures de l'iconographie historique qui consiste à établir une datation en fonction de l'image, elle-même tributaire de la liberté de l'artiste.

3 Après avoir étudié les différentes hypothèses avancées pour l'utilisation des gouaches de Lesueur, Philippe de Carbonnières formule l'hypothèse d'un usage destiné au théâtre, non pas à ces petits théâtres itinérants dont Louis-Sébastien Mercier raconte le fonctionnement dans son Tableau de Paris, qui suppose des déplacements et des 
manipulations (et les gouaches sont fragiles), mais peut-être à un théâtre institutionnalisé, fonctionnant dans une pièce assez grande, pour des représentations peu fréquentes. Le mystère est donc partiellement levé. Enfin, l'examen du choix des scènes représentées fournit un dernier élément à l'enquête iconographique menée par ce livre. On y trouve une attention soutenue aux violences faites en province (on notera la planche 33, "Jourdan dit coupe-tête", qui évoque le massacre de la Glacière à Avignon en septembre 1791) ou à la guerre de Vendée (voir en particulier la planche 36, "L'héroïne de Milhier ", dont la composition présente une très grande similitude avec toutes celles que Claude Langlois a étudiées - Claude Langlois, « Les dérives vendéennes de l'imaginaire révolutionnaire", Annales ESC, mai-juin 1988, n 3, pp. 771-797). Rappelons que la guerre de Vendée a très rarement eu les faveurs de l'iconographie patriote de la première période. La première scène, qui a fait l'objet de gravures polémiques d'origine royaliste, a été également reprise dans L'histoire impartiale de Prudhomme dès 1796, et cette conjonction pourrait renforcer l'hypothèse d'une datation post-thermidorienne formulée par Philippe de Carbonnières.

Cet ouvrage, écrit d'une plume très alerte, se compose d'une partie correspondant aux recherches à proprement parler historiennes de l'auteur (les pages 1 à 62) puis d'une analyse détaillée de chacune de ces gouaches (description, contextualisation, commentaires savants) dont il faut rappeler le caractère esthétique ou le grand intérêt documentaire. 